\title{
Variability of Creatinine Measurements in Clinical Laboratories: Results from the CRIC Study
}

\author{
Marshall Joffe ${ }^{c, d, g}$ Chi-yuan Hsu ${ }^{e}$ Harold I. Feldman ${ }^{\text {b-d, g }}$ \\ Matthew Weir ${ }^{f} \quad$ J.R. Landis ${ }^{c, d, g} \quad$ L. Lee Hamma \\ on behalf of the Chronic Renal Insufficiency Cohort (CRIC) Study Group \\ a Departments of Medicine and Epidemiology and Hypertension and Renal Center, Tulane University, New Orleans, La., \\ ${ }^{b}$ Department of Medicine, ${ }^{c}$ Center for Clinical Epidemiology and Biostatistics, and ${ }^{\mathrm{d} D e p a r t m e n t}$ of Biostatistics and \\ Epidemiology, University of Pennsylvania, Philadelphia, Pa., e Department of Medicine, University of California,

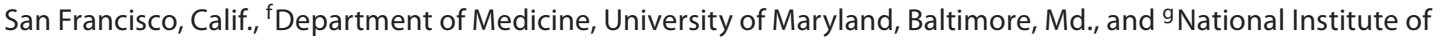 \\ Diabetes and Digestive and Kidney Diseases, National Institutes of Health, Bethesda, Md., USA
}

\section{Key Words}

Chronic renal disease - Creatinine measurements, variability $\cdot$ Chronic Renal Insufficiency Cohort (CRIC) Study $\cdot$ Glomerular filtration rate

\begin{abstract}
Objectives: Estimating equations using serum creatinine $(\mathrm{SCr})$ are often used to assess glomerular filtration rate (GFR). Such creatinine $(\mathrm{Cr})$-based formulae may produce biased estimates of GFR when using Cr measurements that have not been calibrated to reference laboratories. In this paper, we sought to examine the degree of this variation in $\mathrm{Cr}$ assays in several laboratories associated with academic medical centers affiliated with the Chronic Renal Insufficiency Cohort (CRIC) Study; to consider how best to correct for this variation, and to quantify the impact of such corrections on eligibility for participation in CRIC. Variability of $\mathrm{Cr}$ is of particular concern in the conduct of CRIC, a large multicenter study of subjects with chronic renal disease, because eligibility for the study depends on Cr-based assessment of GFR. Methods: A library of 5 large volume plasma specimens from apheresis patients was assembled, representing levels of
\end{abstract}

plasma $\mathrm{Cr}$ from 0.8 to $2.4 \mathrm{mg} / \mathrm{dl}$. Samples from this library were used for measurement of $\mathrm{Cr}$ at each of the $14 \mathrm{CRIC}$ laboratories repetitively over time. We used graphical displays and linear regression methods to examine the variability in $\mathrm{Cr}$, and used linear regression to develop calibration equations. We also examined the impact of the various calibration equations on the proportion of subjects screened as potential participants who were actually eligible for the study. $\boldsymbol{R} \boldsymbol{e}$ sults: There was substantial variability in $\mathrm{Cr}$ assays across laboratories and over time. We developed calibration equations for each laboratory; these equations varied substantially among laboratories and somewhat over time in some laboratories. The laboratory site contributed the most to variability (51\% of the variance unexplained by the specimen) and variation with time accounted for another $15 \%$. In some laboratories, calibration equations resulted in differences in eligibility for CRIC of as much as $20 \%$. Conclusions: The substantial variability in $\mathrm{SCr}$ assays across laboratories necessitates calibration of $\mathrm{SCr}$ measures to a common standard. Failing to do so may substantially affect study eligibility and clinical interpretations when they are determined by Cr-based estimates of GFR.

Copyright $\odot 2010$ S. Karger AG, Basel

\section{KARGER}

두 2010 S. Karger AG, Basel

Fax +41613061234 E-Mail karger@karger.ch www.karger.com www.karger.com/ajn
Lee Hamm, MD

Department of Medicine, SL-12, Tulane University School of Medicine 1430 Tulane Avenue

New Orleans, LA 70112 (USA)

Tel. +1 504988 7800, Fax +1 504988 1600, E-Mail Lhamm@tulane.edu 


\section{Introduction}

Chronic kidney disease (CKD) is recognized to be a significant public health problem as it is common [1] and leads to both end-stage renal disease and excess cardiovascular morbidity and mortality [2]. Serum creatinine (SCr) has been used to assess renal function for decades. In recent years, $\mathrm{SCr}$ values have been extensively used in equations to estimate glomerular filtration rate (GFR). These estimating equations such as the Cockcroft-Gault or Modification of Diet in Renal Disease (MDRD) are practical compared with traditional methods of GFR measurement, which require timed urine collection and are, therefore, much less convenient. These equations (described below) only require $\mathrm{SCr}$ and a few other readily available parameters such as age, gender, ethnicity, and weight.

A major potential problem in the use of these estimating equations is systematic errors associated with the $\mathrm{SCr}$ measurement [3]. Creatinine $(\mathrm{Cr})$ has been recognized to be one of the most variable of routine laboratory tests [4]. Calibration of SCr in most clinical laboratories has not traditionally been standardized to a common gold standard [4]. This variability in $\mathrm{Cr}$ measurements has been known for some time, but has not been fully appreciated and addressed. As discussed below, efforts are currently underway to address this nationally. Some investigators have suggested previously that the variability from laboratory to laboratory is predominantly a fixed 'offset', i.e., the differences between two particular laboratories being relatively constant across a range of SCr from low to high $[5,6]$, but this assumption has not been rigorously tested.

Any measurement error in SCr will translate into errors in estimated GFR (eGFR) when the SCr values are converted to estimated levels of GFR using the MDRD estimating equation or other estimating equations. This will not only result in misclassification of the renal function of individuals, but in errors estimating the population prevalence of CKD in epidemiologic studies [7, 8]. In addition, if uncalibrated SCr-based eGFR were used as the eligibility criteria for any clinical study, selection bias may result.

The present study aims to examine the variability in SCr measurement across 14 participating laboratories from seven clinical sites of the multicenter NIDDK-sponsored Chronic Renal Insufficiency Cohort (CRIC) Study [9]. Our goals were: (1) to document the variation in $\mathrm{Cr}$ assays across the clinical laboratories associated with each of the participating CRIC enrolling sites; (2) to determine the stability of laboratory assays over time; (3) to test whether between-laboratory SCr calibration differ- ences are adequately captured by a 'fixed offset' or whether both slope and intercept corrections are needed (see below); (4) to assess the effect of using uncalibrated SCr data on eligibility for the CRIC Study, and (5) to describe the calibration activities which were ultimately implemented to aid enrollment into the CRIC Study.

\section{Methods}

\section{Setting}

The CRIC Study is an NIH-funded national longitudinal study of renal insufficiency and cardiovascular disease. Eligibility for this cohort study of approximately 3,600 subjects was based largely on criteria regarding age-specific levels of GFR. Specifically, participants aged 21-44 were eligible if their MDRD equation eGFR were $20-70 \mathrm{ml} / \mathrm{min} / 1.73 \mathrm{~m}^{2}$; participants aged $45-64$ were eligible if their MDRD equation eGFR were $20-60 \mathrm{ml} /$ $\mathrm{min} / 1.73 \mathrm{~m}^{2}$ and participants aged $65-74$ were eligible if their MDRD equation eGFR were $20-50 \mathrm{ml} / \mathrm{min} / 1.73 \mathrm{~m}^{2}$. Participants were recruited from 13 discrete clinical sites organized as seven CRIC Clinical Centers across the United States. Although clinical follow-up during the CRIC Study includes some measurement of GFR using the clearance of iothalamate, this evaluation was not practical at the point of screening for entry into the study. Therefore, for the purpose of defining eligibility, GFRs were estimated using a formula derived from the MDRD Study which relies on measured SCr, age, race, and gender [10].

\section{Creatinine Specimens and Assays}

A library of 5 large volume plasma specimens from apheresis patients was assembled, representing levels of plasma $\mathrm{Cr}$ from 0.8 to $2.4 \mathrm{mg} / \mathrm{dl}$. These large volume specimens were used to provide the opportunity to repeat calibration analyses across the 13 CRIC laboratories repetitively over time (1 of which also serves as the core CRIC laboratory). The mean Cr levels for these specimens, as assayed initially at the Cleveland Clinic laboratory, were $0.8,0.9$, $1.74,2.06$, and $2.4 \mathrm{mg} / \mathrm{dl}$. The Cleveland Clinic laboratory was chosen as the 'reference' laboratory because it had served as the central laboratory for the MDRD Study and so the MDRD equation was based on SCr measurements performed at the Cleveland Clinic. For each of the two calibration studies we conducted over time, 5 aliquots of each plasma specimen were sent to each of the clinical laboratory sites, as well as to the Cleveland Clinic laboratory. Thus, each site performed $25 \mathrm{Cr}$ assays for each study which were performed from April to July 2003 and again from September to December 2004. The laboratories used by some of the clinical sites changed over time, thus there are assays from some laboratories from the first time period, but not the second, and vice versa.

\section{Statistical Analysis}

To understand the determinants of the measured level of $\mathrm{Cr}$, we plotted the mean value of $\mathrm{Cr}$ measured from each specimen at the first time against the value for the same specimen measured at the Cleveland Clinic 'reference' laboratory; we also plotted the mean value of $\mathrm{Cr}$ measured from each specimen by time and laboratory at which the assay was performed. To gauge the reproducibility of $\mathrm{Cr}$ measurements at a given time in each laboratory, we 
Fig. 1. Variation in $\mathrm{Cr}$ by site. Each point represents the measured Cr value of a particular plasma sample at the core laboratory (x-axis) and at each one of the other laboratory sites, A-O (y-axis). Each line connects measurements from the same laboratory.

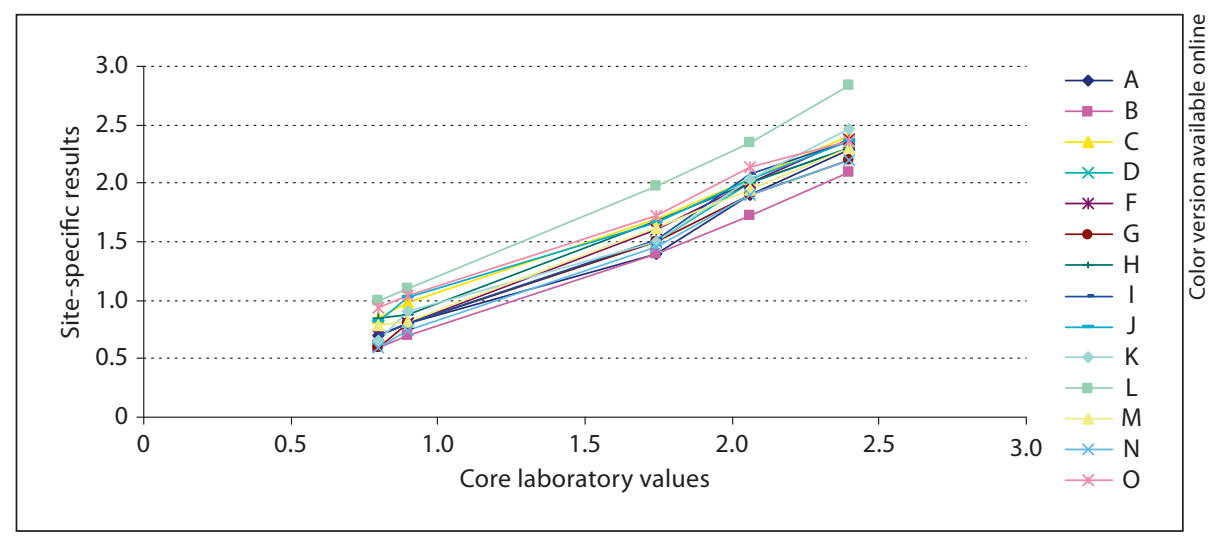

Fig. 2. Mean Cr by specimen, site, and time. Mean Crvalues measured for each of 5 pooled specimens obtained at each laboratory, at each of two times. Measurements from the same specimen at the same site from different periods are connected by a solid line. Points not connected by line segments are from laboratories in which measurements were performed in only one of the two periods.

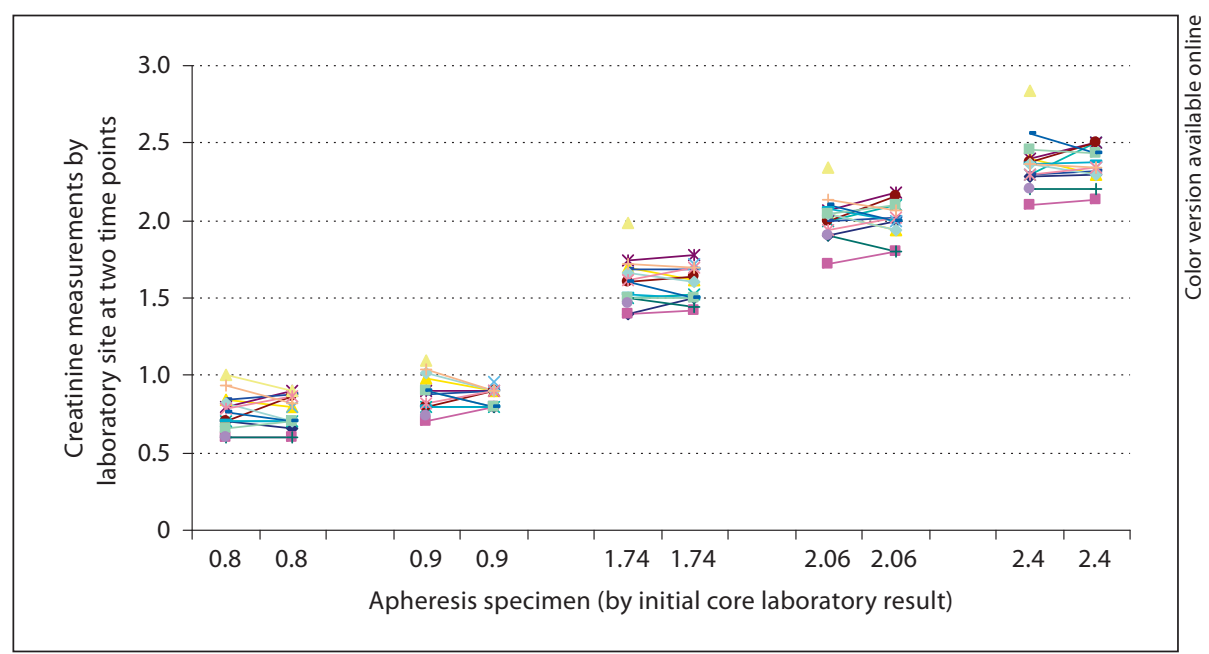

estimated intraclass correlation coefficients for all measurements at that time and laboratory; these coefficients measure the proportion of the variance in $\mathrm{Cr}$ that is due to differences in specimens rather than differences among replicate assays.

To understand the determinants of the measured level of $\mathrm{Cr}$, we plotted the mean value of $\mathrm{Cr}$ measured from each specimen by time and laboratory. To explore more fully the nature of the variability, we performed multiple regression analyses, in which we modeled the $\mathrm{Cr}$ as a function of the specimen, the time, and the laboratory. To allow departures from additivity, we considered terms for the interaction between laboratory and the Cr level measured at the central GFR laboratory, and allowed these associations to change from the first to the second period; we included in this regression only laboratories with measurements in both periods. We used a variance components random effects analysis to assess the degree of variability of measured $\mathrm{Cr}$ associated with each source and report the results in terms of the standard deviation (SD) due to the component.

We then developed site-specific equations calibrating the $\mathrm{Cr}$ measured at each laboratory to the Cr measured and assayed concurrently at the central GFR laboratory. For this purpose, we fit linear regression models, regressing the mean $\mathrm{Cr}$ at the reference laboratory on the individual Cr values from the laboratory of origin; the amount of the correction at any site may vary with the initially measured $\mathrm{Cr}$. We tested whether the regression coefficients were the same at all sites, and whether the regression slopes equaled 1. For comparison, we also fit regression models in which the slopes for each site were fixed to be 1 (i.e., intercept only models); in these latter equations, calibration consists simply of adding or subtracting a constant value for any $\mathrm{Cr}$ derived from the site.

To measure the impact of variability in laboratories on assessment of renal function, we calculated the corrected eGFR for a subject with given levels of SCr measured at their screening laboratory. In addition, we determined the impact of different calibration methods for recruitment into CRIC, by calculating what proportion of screened subjects would have been eligible using calibrated or uncalibrated Crs.

\section{Results}

Figure 1 shows the means of the measurements from each of the different specimens at each of the different sites during the initial calibration. Figure 2 shows the measurements for all sites at both the initial and second calibration time point. The mean intraclass coefficient 
Table 1. Relative contributions of various factors to variability of SCr

\begin{tabular}{|c|c|c|c|c|c|c|c|}
\hline Source & DF & $\begin{array}{l}\text { Sum of } \\
\text { squares } \\
\text { (type I) }\end{array}$ & $\begin{array}{l}\text { Mean } \\
\text { square }\end{array}$ & F value & $\operatorname{Pr}>F$ & $\begin{array}{l}\text { Variance com- } \\
\text { ponent (as stan- } \\
\text { dard deviation) }\end{array}$ & $\begin{array}{l}\text { Proportion of } \\
\text { residual vari- } \\
\text { ance explained }\end{array}$ \\
\hline Specimen & 4 & 251.48 & 62.8701 & $40,655.2$ & $<0.0001$ & 0.697 & \\
\hline Laboratory & 12 & 3.48 & 0.2900 & 187.5 & $<0.0001$ & 0.072 & 0.51 \\
\hline Laboratory $\times$ specimen & 48 & 1.55 & 0.0323 & 20.9 & $<0.0001$ & 0.041 & 0.23 \\
\hline Time (laboratory $\times$ specimen) & 65 & 1.01 & 0.0155 & 10.0 & $<0.0001$ & 0.053 & 0.15 \\
\hline Error & 517 & 0.80 & 0.0015 & & & 0.039 & 0.12 \\
\hline Corrected total & 646 & 258.32 & & & & & \\
\hline
\end{tabular}

Augmented analysis of variance (ANOVA) table for the regression of the individual measurements of $\mathrm{Cr}$ from the pooled specimens on the specimen identifier, laboratory, and time, including the specified interactions, and also the variability due to each of these sources, as well as that due to residual error. The variance component is expressed as a standard deviation and represents the variability in the measured $\mathrm{Cr}$ due to each specified source; the proportion of residual variance explained is the proportion of the total sum of squares not accounted for by specimen $(258.32$ $-251.48=6.84$ ) that is explained by each factor. from each of the different sites and times was 0.996, the largest 0.999 , and the smallest 0.986 , demonstrating that the laboratory measurements at each center at any time were highly reproducible. Several features are notable from figures 1 and 2 . There is substantial variation among the sites in measurements of $\mathrm{Cr}$ at all levels of plasma Cr. Second, there is some drift at many sites over time in the measurement of the same sample. There was no consistent direction to that drift. The variability within sites over time appears less than the variability between sites.

Table 1 shows the results of a formal regression analysis, predicting the measured levels of $\mathrm{SCr}$ as a function of which apheresis specimen was being measured, laboratory at which the assay was performed, and time of measurement. The sums of squares associated with a factor provide a measure of the amount of the total variability in Cr measurement explainable by that factor. Not surprisingly, the true concentration, represented by the specimen, accounts for the largest amount of variability ( $97.4 \%$ of overall variance; SD in $\mathrm{Cr}$ due to site $0.7 \mathrm{mg} / \mathrm{dl}$ ). Overall differences among laboratories provide the next most $(51 \%$ of variance unexplained by specimen; SD 0.07). Additionally, there are laboratory-by-specimen interactions, indicating that the differences among laboratories are not simply a constant shift or offset associated with each laboratory (this is addressed further below). There are also laboratory-by-time and laboratory-byspecimen interactions, indicating that the way individual laboratories measured $\mathrm{Cr}$ changed over time; these account for the smallest amount of variability (sum of squares $15 \%$ of residual variability; SD 0.053). Finally, the
Table 2. Calibration equations for SCr, by site and two time points

\begin{tabular}{|c|c|c|c|c|c|c|}
\hline \multirow{2}{*}{$\begin{array}{l}\text { Labo- } \\
\text { ratory }\end{array}$} & \multicolumn{2}{|c|}{ First period } & \multicolumn{2}{|c|}{ Second period } & \multirow{2}{*}{$\begin{array}{l}\Delta \text { inter- } \\
\text { cept }\end{array}$} & \multirow{2}{*}{$\begin{array}{l}\Delta \\
\text { slope }\end{array}$} \\
\hline & intercept & slope & intercept & slope & & \\
\hline 101 & 0.13 & 1.02 & 0.18 & 1.01 & 0.05 & -0.01 \\
\hline 201 & 0.16 & 1.09 & 0.17 & 1.10 & 0.01 & 0.01 \\
\hline 202 & -0.09 & 1.05 & -0.04 & 1.12 & 0.05 & 0.07 \\
\hline 203 & 0.13 & 0.99 & 0.25 & 0.92 & 0.12 & -0.07 \\
\hline 302 & 0.15 & 0.96 & 0.06 & 0.99 & -0.09 & 0.03 \\
\hline 303 & 0.15 & 1.02 & 0.17 & 1.08 & 0.02 & 0.06 \\
\hline 304 & -0.06 & 1.07 & -0.08 & 1.11 & -0.02 & 0.04 \\
\hline 401 & 0.17 & 0.94 & 0.19 & 0.99 & 0.02 & 0.05 \\
\hline 402 & -0.10 & 1.06 & 0.07 & 1.06 & 0.17 & 0 \\
\hline 403 & 0.18 & 0.92 & 0.24 & 0.94 & 0.06 & 0.02 \\
\hline 501 & -0.06 & 0.88 & & & & \\
\hline 502 & & & -0.06 & 1.09 & & \\
\hline 601 & 0.03 & 1.04 & -0.07 & 1.10 & -0.1 & 0.06 \\
\hline 700 & 0.20 & 1.00 & & & -0.2 & -1 \\
\hline 701 & -0.21 & 1.09 & -0.02 & 1.07 & 0.19 & -0.02 \\
\hline 702 & 0.14 & 0.91 & 0.20 & 0.98 & 0.06 & 0.07 \\
\hline
\end{tabular}

Each intercept-slope pair is obtained by regressing the mean $\mathrm{Cr}$ obtained by regressing the mean $\mathrm{Cr}$ at the reference laboratory on the Crs measured at each of the clinical laboratories.

residual variability is that due to the reproducibility of measurements of the same $\mathrm{Cr}$ at the same time. This is much smaller than the variability due to any of the other factors (12\% of residual variability; SD 0.039), and confirms the earlier results that $\mathrm{Cr}$ measurement at any given time is highly reproducible at the various sites. 
Fig. 3. Differences between core laboratory results and results at each site. Each point represents the site-specific measurement minus the core laboratory result. Lines connect results at each specific site. Perfectly horizontal lines would indicate a constant difference (intercept) between measurements across the range of Crs (slope $=1$ ). That most laboratories (and lines) vary significantly from horizontal indicates that slopes as well as intercepts have to be considered as indicated in the text.

Fig. 4. a The difference between two calibrated values of SCr: the fixed-slope calibration and the full calibration. $\mathbf{b}$ The difference between the uncalibrated $\mathrm{Cr}$ and the full calibration. On the whole, the differences between the calibrated values are substantially less than the differences between calibrated and uncalibrated Cr. Nonetheless, the difference between the different calibrations is sometimes substantial, especially at higher levels of $\mathrm{Cr}$ (beyond the range of Crs in the 5 pooled specimens). Even in the lower range of SCr, differences between the calibrations could be larger than 0.1 unit.
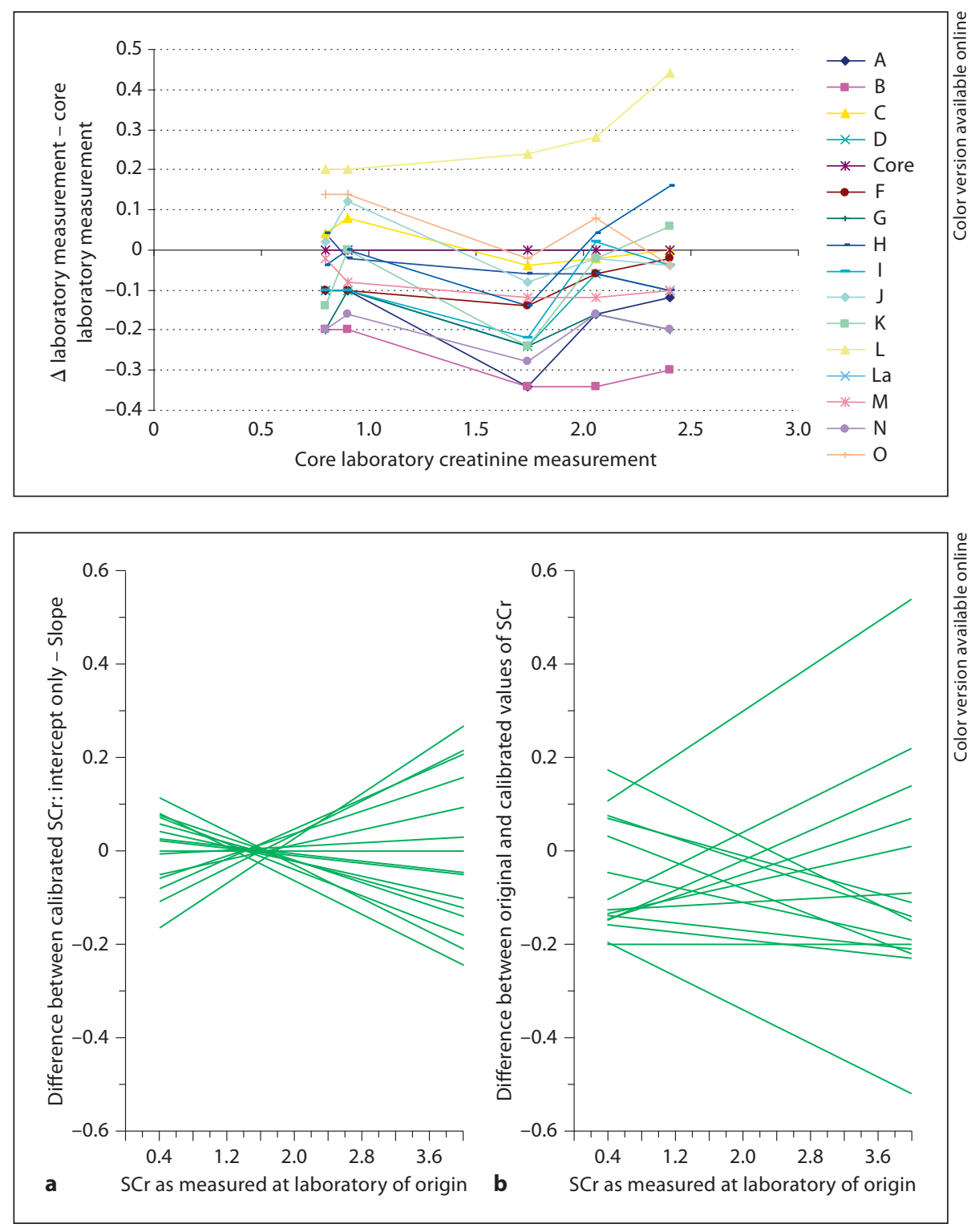

All of this supports the necessity of calibrating $\mathrm{Cr}$ readings from various sites to a common standard. To do this, we performed least squares regressions, regressing the $\mathrm{Cr}$ measured at the Cleveland Clinic reference core laboratory on the $\mathrm{Cr}$ measured at each individual laboratory. Table 2 presents slopes and intercepts from two such regressions - the first is based on the assays performed during the first calibration study and the second is based on assays performed during the second calibration study. Some sites vary very little, others vary moderately over time; in no case did recalibration cause either slope or intercept to vary across most of the range of the slopes and intercepts. Both intercept and slope vary from labo- ratory to laboratory. In these studies, variations in both intercept and slope were found to be important as illustrated in figures 3 and 4 . This argues that calibration can be improved by estimating slopes as well as intercepts, contrary to previous studies $[5,6]$. Figure 3 illustrates the difference between individual laboratory results and the core laboratory across the range of $\mathrm{Crs}$ in the specimens tested. Depicted in this way, if slopes were essentially equal to 1 , then each of the lines would be flat or horizontal, which most of the lines are not. Figure 4 illustrates this in a more formal way: figure 4 a shows the difference between fully calibrated Cr (using both intercept and slope) and Cr calibrated using only an intercept and a 


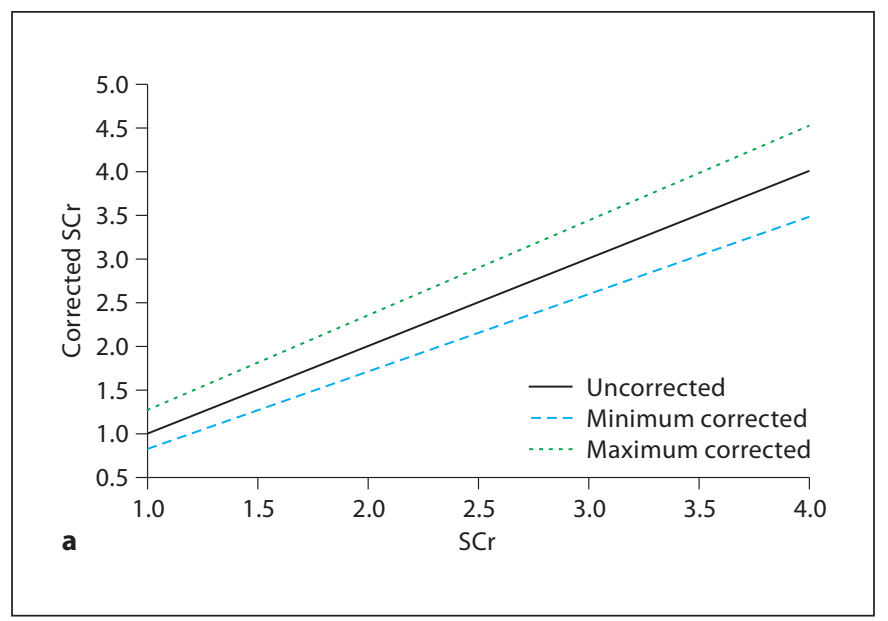

Fig. 5. Range of calibration corrections to SCr and eGFR. a The range of calibration-corrected values for a $\mathrm{SCr}$ measurement made at an individual laboratory. The $\mathrm{x}$-axis shows the initial, uncorrected value of the SCr measured at a given laboratory. The $\mathrm{y}$-axis plots the smallest or largest value of $\mathrm{Cr}$ that would result from a correction based on the laboratory-specific calibration equations in table 2. Thus, an uncorrected $\mathrm{Cr}$ of 2.0 from one (extreme) laboratory would be adjusted down to 1.7 , and from another (opposite extreme) laboratory would be adjusted up to 2.3.

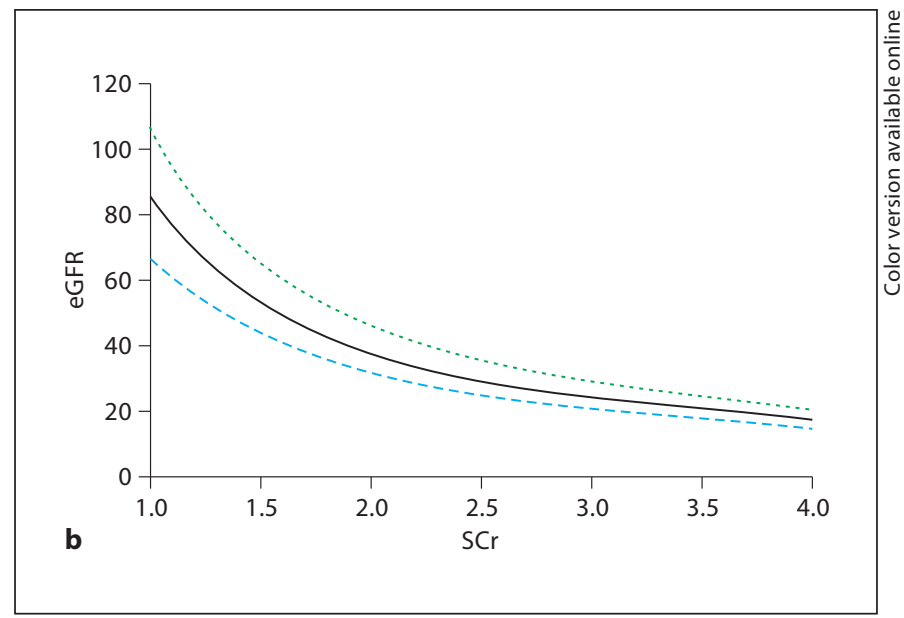

b The range of calibration-corrected values for eGFR based on a SCr measurement made at an individual laboratory. The eGFR is for a 45-year-old white male. The $\mathrm{x}$-axis shows the initial, uncorrected value of the SCr measured at a given laboratory. The y-axis plots the smallest or largest value of eGFR that would result from a correction. Thus, an uncorrected Cr of 1.0 from one (extreme) laboratory would correspond to a corrected eGFR of 67, and that from another (opposite extreme) laboratory would correspond to a corrected eGFR of 107.

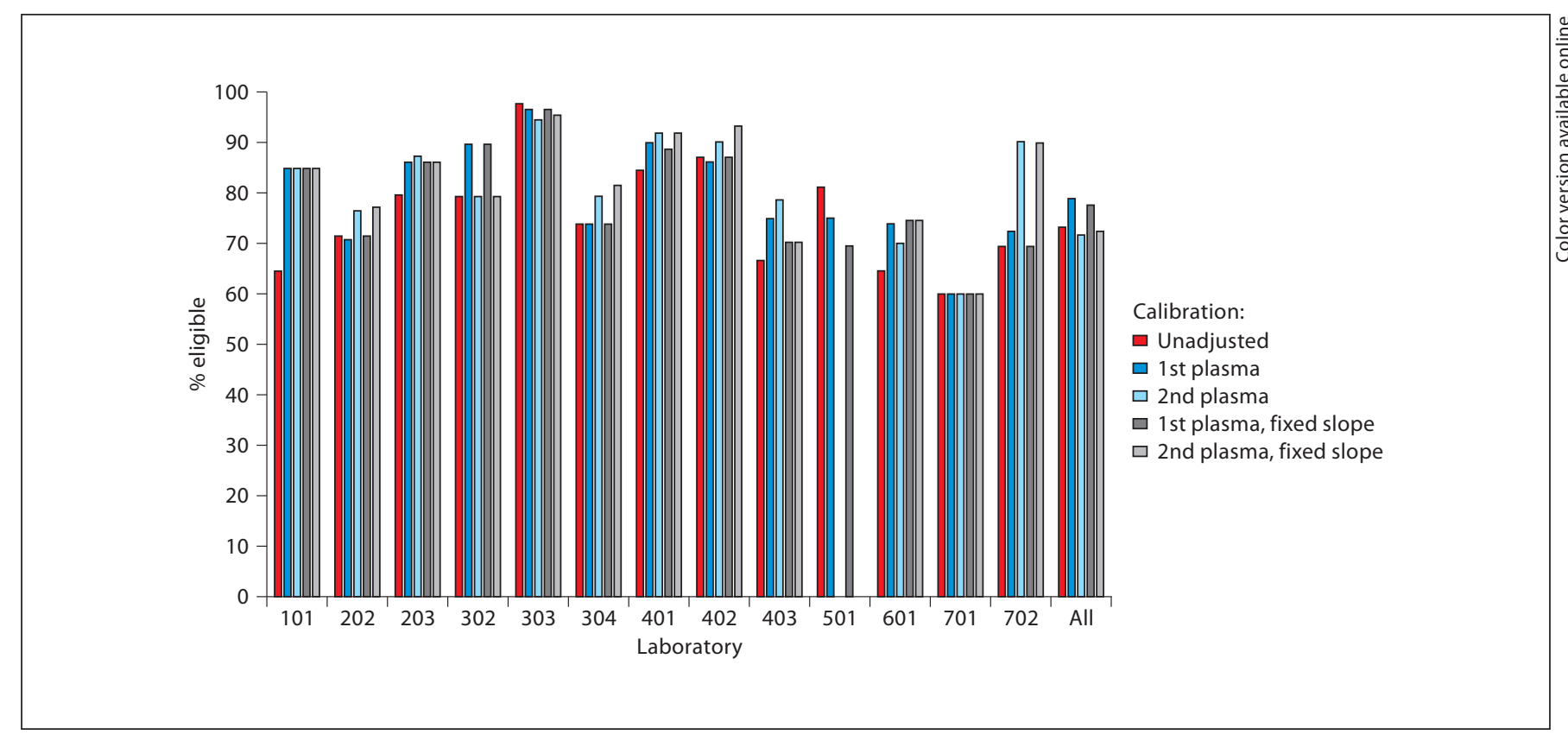

Fig. 6. Proportion eligible for inclusion in CRIC, by laboratory criterion. This figure shows the proportion of subjects deemed eligible for inclusion in CRIC, by laboratory of origin, and accord- ing to whether eligibility is based on uncalibrated eGFR, or eGFR based on the first or second calibration equations, or using a fixed-slope method as described in the text. 
fixed slope (fixed-slope calibration). Using only intercept results in substantial variation from fully calibrated values, especially at high values of SCr. Figure $4 \mathrm{~b}$ demonstrates the difference between fully calibrated and uncalibrated values. On the whole, the differences between the calibrated values are substantially less than the differences between calibrated and uncalibrated $\mathrm{Cr}$.

We considered the consequences of different calibration methods for measurement of SCr and eGFR. Figure 5a shows how a $\mathrm{SCr}$ at a given laboratory translates into corrected or calibrated $\mathrm{Cr}$; for example, a subject with a $\mathrm{Cr}$ of 2.0 could have a calibrated $\mathrm{Cr}$ as low as 1.7 (if the original assay were done at one site) or as high as 2.3 (were the original assay done at another). Although overall variability increases with higher $\mathrm{Cr}$, the relative variability declines somewhat, i.e., measurement of higher levels of $\mathrm{Cr}$ is somewhat less variable as a percent of the absolute value. For instance, at a $\mathrm{Cr}$ of 1.0 , the range is $0.83-1.25$ or $42 \%(0.42 / 1.0=42 \%)$, whereas at 4.0 , the range is $3.48-4.52$ or only $26 \%(1.04 / 4=26 \%)$. Figure $5 b$ shows how different serum values of uncalibrated $\mathrm{SCr}$ translate into eGFR (a hypothetical 45-year-old white male is used in the illustration). This dramatically demonstrates how variability in $\mathrm{Cr}$ measurement at lower values (e.g. SCr in the 1.0-2.0 range) has the expected greater effect on eGFR variation than at higher SCrs (e.g. $>3$ ).

Finally, we considered how different calibration methods affect eligibility into the CRIC Study. Figure 6 shows the proportion of subjects screened at each site who were deemed eligible for inclusion in CRIC, using eGFRs based on different versions of SCr (fully calibrated and fixedslope calibrated values from both periods, and uncalibrated values). The proportion varied substantially by site, from 60 to $>90 \%$; this reflects in part the different populations screened at the various sites. In addition, the proportion deemed eligible by various criteria sometimes varied substantially, depending on which calibration was used. For example, at site 101, only $64.6 \%$ of subjects would be deemed eligible without calibrating $\mathrm{Cr}$, whereas $84.7 \%$ were eligible using the first calibration coefficients. The differences between the fixed-slope calibrations and full calibrations were largest for laboratories in which the slopes differed substantially from 1; these differences were, on the whole, smaller than differences between calibrated and uncalibrated Crs. Ultimately, in CRIC, we determined enrollment eligibility based on eGFR using calibrated SCr measurements obtained locally at each enrolling clinical site and regression equations including both slope and intercept terms, which were updated over time during the window of enrollment.

\section{Discussion}

CKD is recognized to be a major public health problem leading to both end-stage renal disease and premature cardiovascular disease. The most common method to currently diagnose CKD is measurement of $\mathrm{SCr}$ and subsequent estimation of GFR [11]. Because of the convenience and overall accuracy of estimating equations, many clinical laboratories started reporting eGFR before $\mathrm{SCr}$ calibration issues were fully addressed. The potential inaccuracy in eGFR due to calibration errors in Cr measurement has not been sufficiently appreciated in the past. This study demonstrates significant variability in SCr determinations in clinical laboratories associated with academic medical centers prior to the start of the CRIC Study recruitment in 2002. This variability has significant clinical implications and also substantial potential to affect clinical research studies whose enrollment of participants is dependent on Cr-based measures of eGFR.

In the present study, this variability was more pronounced across centers than over time, and differed depending on the absolute value of the $\mathrm{Cr}$ concentration (variation in both slope and intercept of the correlation relationship). As expected, based on the nature of the relationship between SCr and eGFR, this variability particularly affects eGFR at higher eGFR values (lower $\mathrm{Cr}$ ) as previously reported [6].

Since our analysis is based on a 'split sample' approach, observed differences in $\mathrm{Cr}$ measurement cannot be due to changes in renal function or other biological causes, but rather are due to assay measurement issues. Measurement variation of $\mathrm{Cr}$ occurs based on machine type and manufacturer, method, and calibration to standards [12, 13]. College of American Pathologists surveys have shown that calibration 'bias' in SCr measurements is very common, and in fact SCr was the analyte most frequently showing a significant calibration bias among routine chemistry panels $[4,14]$.

The impact of these calibration differences in $\mathrm{Cr}$ on eGFR is potentially significant. This will be particularly true when trying to distinguish variations in eGFR values from two different laboratories or when trying to classify the stage of kidney disease. Unless properly calibrated, relatively low SCrs (e.g. $1.2-1.5 \mathrm{mg} / \mathrm{dl}$ ) might reflect either a normal GFR (i.e. $>60 \mathrm{ml} / \mathrm{min} / 1.73 \mathrm{~m}^{2}$ in the absence of other evidence of kidney disease such as proteinuria) or stage 3 CKD (in which eGFR is $<60 \mathrm{ml} / \mathrm{min} / 1.73 \mathrm{~m}^{2}$ ). For instance, for a 45 -year-old white male with unadjusted $\mathrm{Cr}$ of 1.3 at each laboratory, the range of adjusted $\mathrm{Cr}$ was $1.09-1.58 \mathrm{mg} / \mathrm{dl}$, yielding a range of eGFR of 50.90-77.75 
$\mathrm{ml} / \mathrm{min} / 1.73 \mathrm{~m}^{2}$. This can have not only clinical implications but also perhaps financial implications (e.g. insurance billing). The variations in measured $\mathrm{Cr}$ have significantly more relative effect at lower $\mathrm{Cr}$ values (higher GFRs) than at higher Cr values (lower GFR) (fig. 5).

The present study has several strengths compared to other reports. First, actual specimens with a range of absolute Cr levels, rather than pooled data or specimens [15] or simulations [16], were used. Also, more recent samples rather than remote studies were used. In addition, the present analysis provides concrete illustration of the effect of variations in measured Cr on eGFR and directly impacted enrollment into a large, high-profile $\mathrm{NIH}$ sponsored CRIC Study.

The present study also has several limitations. First, only a limited number of laboratories were involved. However, these laboratories were all associated with academic medical centers and, hence, might be presumed to be at least as rigorous as clinical laboratories in general. Second, the use of plasma samples differs from the usual measurement of serum specimens; this presumably has minimal but unknown effects. The use of apheresis samples allowed the acquisition of large volumes which could be distributed to a number of laboratories and the ability to use the exact same sample over time.

This study highlights the importance of uniform standardization of the measurement of SCr across clinical laboratories and ongoing efforts like those led by the Laboratory Working Group of the National Kidney Disease Education Program (NKDEP) for standardization and improved accuracy of SCr measurements in clinical laboratories [17]. Under this effort, $\mathrm{Cr}$ reference materials will be traceable to primary reference material at the National Institutes of Standards and Technology (NIST), with assigned values traceable to isotope dilution mass spectrometry. As this plan is implemented, clinicians will need to recognize the impact on serial measured $\mathrm{Cr}$ (possible shifts in SCr due simply to laboratory calibration rather than to clinical change) and the necessity of this calibration (which will cause a calibration shift in many laboratories from prior historical values). Our data also emphasize the potential value of expanding similar Cr standardization efforts to countries outside of the United States.

Calibration of $\mathrm{Cr}$ is also crucial to ensuring standardized inclusion criteria for multicenter studies utilizing local laboratories for screening. The results here demonstrate that for multicenter studies, calibrated $\mathrm{Cr}$ values are needed if GFR is to be estimated based on formulae derived from a central laboratory. This was anticipated for the CRIC Study, and this anticipation initiated the present calibration studies. The present study demonstrates that calibration should include slopes as well as intercepts in developing equations to transform values to those of central laboratories in contrast to some prior recommendations.

In summary, we have documented substantial variation in $\mathrm{Cr}$ assays across clinical laboratories prior to the start of recruitment. We also determined that variations in Cr measurements occurred within the same laboratories across time (albeit to a lesser degree than that across different laboratories). We found that both slope and intercept corrections need to be taken into consideration for optimal calibration and this was the approach that was ultimately adopted in CRIC to aid enrollment and minimize opportunities for selection bias. Our studies strongly support the current plans underway to standardize SCr measurements and the use of these with appropriate matched equations $[18,19]$.

\section{Acknowledgements}

In addition to funding under a cooperative agreement from National Institute of Diabetes and Digestive and Kidney Diseases (5U01DK060990, 5U01DK060984, 5U01DK06102, 5U01DK061021,5U01DK061028,5U01DK60980,5U01DK060963, and 5U01DK060902), this work was supported in part by the following institutional Clinical Translational Science Awards and other National Institutes of Health grants: Johns Hopkins University UL1 RR-025005, University of Maryland GRCR M01 RR16500, Case Western Reserve University Clinical and Translational Science Collaborative (University Hospitals of Cleveland, Cleveland Clinic Foundation, and MetroHealth) UL1 RR-024989, University of Michigan GCRC M01 RR-000042 and CTSA UL1 RR-024986, University of Illinois at Chicago Clinical Research Center, M01RR-013987-06, Tulane/LSU/Charity Hospital General Clinical Research Center RR-05096, and Kaiser NIH/NCRR UCSF-CTSI UL1 RR-024131 and 5K24DK002651. Additional support provided by the National Center for Minority Health and Health Disparities, National Institutes of Health, and Department of Veterans Affairs.

References

1 Coresh J, Selvin E, Stevens LA, et al: Prevalence of chronic kidney disease in the United States. JAMA 2007;298:2038-2047.

2 Go AS, Chertow GM, Fan D, McCulloch CE, Hsu CY: Chronic kidney disease and the risks of death, cardiovascular events, and hospitalization. N Engl J Med 2004;351: 1296-1305.

3 Hsu CY, Chertow GM, Curhan GC: Methodological issues in studying the epidemiology of mild to moderate chronic renal insufficiency. Kidney Int 2002;61:1567-1576. 
-4 Ross JW, Miller WG, Myers GL, Praestgaard $\mathrm{J}$ : The accuracy of laboratory measurements in clinical chemistry: a study of 11 routine chemistry analytes in the College of American Pathologists Chemistry Survey with fresh-frozen serum, definitive methods, and reference methods. Arch Pathol Lab Med 1998;122:587-608.

5 Coresh J, Toto RD, Kirk KA, et al: Creatinine clearance as a measure of GFR in screenees for the African-American Study of Kidney Disease and Hypertension pilot study. Am J Kidney Dis 1998;32:32-42.

6 Coresh J, Astor BC, Greene T, Eknoyan G, Levey AS: Prevalence of chronic kidney disease and decreased kidney function in the adult US population: Third National Health and Nutrition Examination Survey. Am J Kidney Dis 2003;41:1-12.

$\checkmark 7$ Clase CM, Garg AX, Kiberd BA: Prevalence of low glomerular filtration rate in nondiabetic Americans: Third National Health and Nutrition Examination Survey NHANES III). J Am Soc Nephrol 2002;13:1338-1349.

$>8$ Coresh J, Eknoyan G, Levey AS: Estimating the prevalence of low glomerular filtration rate requires attention to the creatinine assay calibration. J Am Soc Nephrol 2002;13:28112812 . $\checkmark 9$ Feldman HI, Appel LJ, Chertow GM, et al: The Chronic Renal Insufficiency Cohor (CRIC) Study: design and methods. J Am Soc Nephrol 2003;14:S148-S153.

10 Levey AS, Bosch JP, Lewis JB, Greene T, Rogers N, Roth D: A more accurate method to estimate glomerular filtration rate from serum creatinine: a new prediction equation. Modification of Diet in Renal Disease Study Group. Ann Intern Med 1999;130:461-470.

11 Stevens LA, Coresh J, Greene T, Levey AS: Assessing kidney function-measured and estimated glomerular filtration rate. $\mathrm{N}$ Engl J Med 2006;354:2473-2483.

12 Miller WG, Myers GL, Ashwood ER, et al: Creatinine measurement: state of the art in accuracy and interlaboratory harmonization. Arch Pathol Lab Med 2005;129:297304.

13 Verhave JC, Fesler P, Ribstein J, du Cailar G, Mimran A: Estimation of renal function in subjects with normal serum creatinine levels: influence of age and body mass index. Am J Kidney Dis 2005;46:233-241.

14 Coresh J, Astor BC, McQuillan G, et al: Calibration and random variation of the serum creatinine assay as critical elements of using equations to estimate glomerular filtration rate. Am J Kidney Dis 2002;39:920-929.
15 Stevens LA, Manzi J, Levey AS, et al: Impact of creatinine calibration on performance of GFR estimating equations in a pooled individual patient database. Am J Kidney Dis 2007;50:21-35.

16 Murthy K, Stevens LA, Stark PC, Levey AS: Variation in the serum creatinine assay calibration: a practical application to glomerular filtration rate estimation. Kidney Int 2005; 68:1884-1887.

17 Myers GL, Miller WG, Coresh J, et al: Recommendations for improving serum creatinine measurement: a report from the Laboratory Working Group of the National Kidney Disease Education Program. Clin Chem 2006;52:5-18.

18 Levey AS, Coresh J, Greene T, et al: Using standardized serum creatinine values in the modification of diet in renal disease study equation for estimating glomerular filtration rate. Ann Intern Med 2006;145:247254.

19 Levey AS, Coresh J, Greene T, et al: Expressing the Modification of Diet in Renal Disease Study equation for estimating glomerular filtration rate with standardized serum creatinine values. Clin Chem 2007;53:766-772. 